\section{Neutron Activation Analysis using an Internal Standard}

To correct for differences in neutron flux and neutron shadowing between sample and reference a method has been developed which uses an internal standard.

Instead of irradiating a known amount of the element as a reference, two quantities $a$ and $b$ of the sample are irradiated, an amount $c$ of the element $X$ to be determined being mixed homogeneously with $b$.

The material subjected to analysis should contain at least one element $Y$, which gives rise to an isotope measurable with good accuracy and having a halflife long in comparison with the isotope of the species analysed.

When both samples are measured at a time $t$, after the complete decay of the species due to $X$, the ratio of the activities due to $Y$ will be given by

$$
\frac{A_{t(Y)}}{A_{t(Y)}^{\prime}}=\frac{a}{b} \cdot \alpha
$$

$\alpha$ being a correction factor which compensates for any difference in the neutron flux received by the two samples and/or for a different neutron shadowing.

By analysis of the decay curves, the activities $A_{\tau(X)}$ and $A^{\prime}{ }_{r(X)}$, due to the element $X$, can be computed at the end of the irradiation time $\tau$.

The specific activity of the sample, due to $X$, will be given by :

$$
S_{\imath(X)}=\frac{1}{c}\left[A^{\prime}{ }_{\gamma(X)} \propto-\frac{b}{a} A_{\tau(X)}\right]
$$

and the percentage of $X$ present by:

$$
\% X=\frac{A_{\tau(X)} \times 100}{a \times S_{\tau(X)}}
$$

It is also apparent that simultaneous irradiation of both samples is not required.

The method is of general use but is likely to be more important in the case of neutron sources of low intensity. In this case neutron-shadowing will be greater, as it is necessary to take large samples to reach a sufficiently high activity of the analysed element. Even when using the $B R \cdot I$ reactor, however, operating at $10^{11} \mathrm{n} / \mathrm{cm} .^{2}$ sec., neutron shadowing was observed, causing the specific activity to be low by 15 per cent when irradiating $1-\mathrm{gm}$. samples of high-alloy cobalt steel.

The method was successfully applied in the determination of vanadium and tungsten in high-alloy steels and will be described in detail elsewhere.

$$
\begin{aligned}
& \text { G. Leliafrt } \\
& \text { J. Hoste } \\
& \text { Z. EeckHaUt }
\end{aligned}
$$

Laboratory for Analytical Chemistry, University of Ghent.

$$
\text { June } 23 .
$$

\section{Error at a Rectangular Corner in Relaxation Methods}

IN "Relaxation Methods in Theoretical Physics" (R. V. Southwell, Clarendon Press, 1946, p. 133) results obtained by $R$. W. G. Gandy, using the method of relaxation, are given for the capacity $C$, per unit length, of a cylindrical condenser, the crosssection of which consists of concentric squares having sides in the ratio $2 \sqrt{ } 2: 1$. Three cases are illustrated (Fig. 54): (a) the two squares are diamondwise to each other ; $(c)$ the sides are parallel; $(b)$ an intermediate position.

It is with cases $(a)$ and $(c)$ that we are here concerned. We have calculated the value of $C$ in these two cases from the analytical solution given by F. Bowman", using Milne-Thomson's "Table of Elliptic Functions".

In case $(a)$ we obtain the value $6 \cdot 630$ for $4 \pi C$, and in case $(c)$ the value $6 \cdot 596$, as compared with Gandy's result of $6 \cdot 715$ in both cases.

H. Jeffreys and B. S. Jeffreys ${ }^{2}$ give, for case (c) with sides in the ratio $2: 1$, results obtained analytically and by relaxation. For this ratio of sides we have computed $4 \pi C$ for case $(a)$ and obtain $10 \cdot 660$, but no corresponding result obtained by relaxation appears to be available.

These results may be of interest to those using relaxation methods, as giving some idea of the amount of error that may be expected at a rectangular corner.

\section{R. Buther}

S. V. FAGG

Department of Mathematics,

Manchester College of Science and Technology. June 17.

1 Bowman, F., Proc. London Math. Soc., 39, 211 (1935)) ; 41, 271 (1936). 2 Jeffreys, H., and Jeffreys, B. S., "Methods of Mathematical Physics", 3rd edit., 312 (Cambridge Univ. Press, 1956).

\section{Two- and Three-Dimensional Flow of Grains through Apertures}

IN the course of experiments on the physics of the granular mass, the rate of discharge of three size grades of a dry, sharp sand was measured $(a)$ from wide cylindrical vessels through circular and elliptical orifices, (b) from wide rectangular vessels through long slits. In both cases the apertures were situated on the central axis of a horizontal base. The mean particle sizes $P$ of the size grades $(18 \times 36,36 \times 60$, $44 \times 120$ British Standard mesh) were $0.053,0.037$, $0.020 \mathrm{~cm}$. From the measured rate of discharge $Q \mathrm{gm} . / \mathrm{sec}$, a mean linear velocity $v \mathrm{~cm}$. $/ \mathrm{sec}$. was calculated as :

$$
v=Q / \rho A
$$

$A$ cm. ${ }^{2}$ being the area of the aperture and $\rho$ the particle density in $\mathrm{gm} . / \mathrm{cm}^{3}$. To find the true linear velocity of the particles from $v$ it would be necessary to measure the bulk density of the flowing material at the orifice; this has not been done.

The data for the three-dimensional flow systems of experiment $(a)$ were found to be well correlated in a plot of the dimensionless magnitudes,

$$
\varphi=\frac{v}{\sqrt{g H}} \text { and }\left(\frac{P}{H}\right)
$$

where $g \mathrm{~cm} . / \mathrm{sec} .{ }^{2}$ is acceleration due to gravity and $H$ is the perimetral diameter of the aperture, defined as 4 (area)/perimeter. The elliptical apertures had equal areas of $0.32 \mathrm{~cm} .{ }^{2}$ and eccentricity increasing from zero to 0.995 : the velocity $v$ decreased with increasing eccentricity. Representation (2) was adequate for accounting for aperture shape. As earlier workers have shown, this representation has the advantage that $\varphi$, which depends essentially on the aperture and not the particles, does not vary much with $H, Q$ being nearly proportional to $H^{2-5}$ for flow through circular apertures. Fowler and 